\title{
Refractory Myelodysplastic Syndrome
}

National Cancer Institute

\section{Source}

National Cancer Institute. Refractory Myelodysplastic Syndrome. NCI Thesaurus. Code C148362.

Myelodysplastic syndrome that does not respond to treatment. 